\title{
Pair Tunneling Resonance in the Single-Electron Transport Regime
}

\author{
M. Leijnse, ${ }^{1,3}$ M. R. Wegewijs, ${ }^{1,2,3}$ and M. H. Hettler ${ }^{4}$ \\ ${ }^{1}$ Institut für Theoretische Physik A, RWTH Aachen, 52056 Aachen, Germany \\ ${ }^{2}$ Institut für Festkörper-Forschung-Theorie 3, Forschungszentrum Jülich, 52425 Jülich, Germany \\ ${ }^{3}$ JARA-FIT, Juelich-Aachen Research Alliance for Future Information Technologies \\ ${ }^{4}$ Institut für Nanotechnologie, Forschungszentrum Karlsruhe, Postfach 3640, 76021 Karlsruhe, Germany
}

(Received 20 March 2009; published 7 October 2009)

\begin{abstract}
We predict a new electron pair tunneling (PT) resonance in nonlinear transport through quantum dots with positive charging energies exceeding the broadening due to thermal and quantum fluctuations. The PT resonance shows up in the single-electron transport (SET) regime as a peak in the derivative of the nonlinear conductance, $d^{2} I / d V^{2}$, when the electrochemical potential of one electrode matches the average of two subsequent charge addition energies. For a single level quantum dot (Anderson model) we find the analytic peak shape and the dependence on temperature, magnetic field, and junction asymmetry and compare with the inelastic cotunneling peak which is of the same order of magnitude. In experimental transport spectroscopy the PT resonance may be mistaken for a weak SET resonance judging only by the voltage dependence of its position. Our results provide essential clues to avoid such erroneous interpretation.
\end{abstract}

Introduction.-Electron tunneling spectroscopy has nowadays become a standard tool for investigating the in situ properties of single-electron transistors based on quantum dots in semiconductor heterostructures, nanowires, carbon nanotubes, and even single molecules. The basic spectroscopy rules derive from the simple energy resonance conditions for single-electron tunneling (SET) onto the dot. As a result, the bias positions of differential conductance $(d I / d V)$ resonances depend linearly on the gate voltage due to capacitive effects [1]. In addition, inelastic cotunneling (COT) processes can often be distinguished as steps in $d I / d V$ [2], allowing for spectroscopy of quantum dot excitations with increased energy resolution [3]. Here an electron is transferred from the high to the low biased electrode through the dot, using the excess biasenergy to excite the dot by an energy $\Delta \epsilon$. Since these electron-hole charge transfer resonances involve only a virtual charging of the dot, they appear at a bias threshold $V=\Delta \epsilon$, independent of the gate voltage. These processes arise only in second order perturbation theory in the tunnel rate $\Gamma$.

In this Letter we show that, in the same order of $\Gamma$, electron pair tunneling (PT) gives rise to distinct, measurable transport effects which, to our knowledge, have been overlooked so far. In a single coherent process, a pair of electrons is extracted from (added to) one electrode, resulting in a real (dis)charging of the dot by two electrons, involving a positive charging energy much larger than broadening due to thermal and quantum charge fluctuations. The corresponding PT resonance appears deep inside the SET region where a finite current is flowing and its position has the same gate-dependence as SET resonances. It might thus be mistaken for a weak SET resonance belonging to some excited state and one might thereby extract an erroneous level-structure from the spectroscopic data. We show how the PT resonance can be distinguished from other types of first and second order processes and what additional information can be extracted from it. Since spectroscopic data obtained from single-electron transistor devices are often nontrivial to interpret we provide several independent criteria for its experimental identification, namely the resonance shape and the dependence on temperature, magnetic field, and junction asymmetry. The PT processes discussed here completely lack any signature inside the Coulomb blockade region where SET is exponentially suppressed. In this region a different type of pair tunneling resonance occurs which is much weaker than the one discussed here since it appears only in the third order in the tunnel rate $\Gamma$ [4]. Also the latter pair tunneling process involves neither real nor virtually doubly occupied states (i.e., it remains finite in the $U=\infty$ limit). Second order pair tunneling resonances were discussed previously for the rare case of negative Coulomb charging energy (i.e., effective attractive electron-electron interaction) [5], related to pair tunneling in superconducting grains [6], where SET is suppressed by the superconducting gap. Also Kondo effect involving electron pairs have been discussed in this limit [7]. Here we consider the experimentally most frequently occurring case of strong positive charging energy.

Model.-The pair tunneling resonance is already present in the generic model for a quantum dot, i.e., the nonequilibrium Anderson model. Here the quantum dot, described by the Hamiltonian $H=\sum_{\sigma} \epsilon_{\sigma} \hat{n}_{\sigma}+U \hat{n}_{\uparrow} \hat{n}_{\downarrow}$, consists of a single orbital with energy $\epsilon_{\sigma}$ and occupation $\hat{n}_{\sigma}=d_{\sigma}^{\dagger} d_{\sigma}$. Here $\epsilon_{\downarrow}-\epsilon_{\uparrow}=h$ equals the Zeeman energy and $U$ is the finite positive charging energy. We let $N=\sum_{\sigma} \hat{n}_{\sigma}$ denote the electron number on the dot. The many-body eigenstates are $|0\rangle,|\sigma\rangle$ and $|2\rangle$ with energies $E_{0}=0, E_{\sigma}=\epsilon_{\sigma}$ and $E_{2}=\sum_{\sigma} \epsilon_{\sigma}+U$. The dot is coupled by a tunneling 
Hamiltonian, $H_{T}=\sum_{r=L, R} \sum_{k, \sigma} T_{r} d_{\sigma}^{\dagger} c_{r k \sigma}+$ H.c., to macroscopically large reservoirs, described by $H_{R}=$ $\sum_{r=L, R} \sum_{k, \sigma} \epsilon_{k} c_{r k \sigma}^{\dagger} c_{r k \sigma}$. The electrons in the reservoirs are assumed to be noninteracting, with operators $c_{r k \sigma}^{\dagger}$, $c_{r k \sigma}$ for state $k$ and spin $\sigma$ in electrode $r=L, R$. The tunnel amplitudes can be expressed in the tunnel rates $\Gamma_{r}$ through $T_{r}=\sqrt{\Gamma_{r} / 2 \pi}$. Throughout the Letter we use natural units where $\hbar=k_{B}=|e|=1$, where $-|e|$ is the electron charge.

Let us first present the basic physics. Figure 1 shows a schematic comparison of tunneling processes for the spindegenerate Anderson model when $E_{\sigma}$ is larger than the average chemical potential $\mu$ of the reservoirs, such that the quantum dot is unoccupied at zero bias voltage, $V=0$. We take $\mu=0$ and assume symmetric biasing such that $\mu_{L}=V / 2, \mu_{R}=-V / 2$. At low bias transport is dominated by cotunneling involving virtual occupation of state $|\sigma\rangle$. For larger bias, $|V / 2|>E_{\sigma}-E_{0}$, electrons can sequentially tunnel into and out of the dot, involving real occupation of state $|\sigma\rangle$. In this regime double occupation of the dot through two consecutive SET processes only becomes energetically allowed when additionally the energy difference $E_{2}-E_{\sigma}$ is below the chemical potential of one lead, i.e., when $|V / 2|>E_{\sigma}+U$. Midway between these resonances the coherent tunneling of a pair of electrons from the same reservoir becomes possible, i.e., at the resonance condition:

$$
|V / 2|>\frac{1}{2}\left(\sum_{\sigma} E_{\sigma}+U\right)
$$

The corresponding process is shown in Fig. 1(c). One can think of this as one electron from just below the Fermi level of the left reservoir tunneling onto state $|\sigma\rangle$, leaving an excess energy $\Delta=U / 2$, which can be used to assist the second electron in reaching state $|2\rangle$. Thus, the total PT process is energy-conserving. However, since there are no internal degrees of freedom on the dot to store the energy $\Delta$, these two processes have to take place coherently in the short time set by the time-energy uncertainty relation.
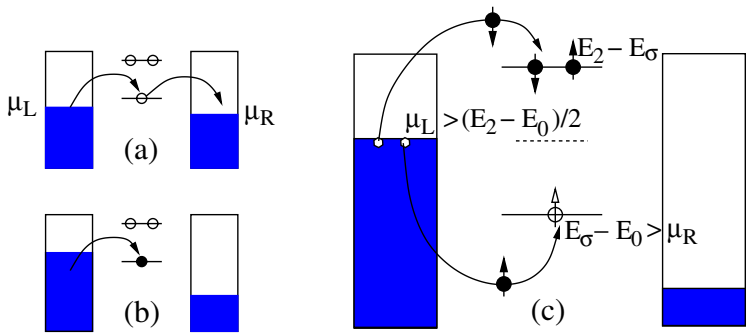

FIG. 1 (color online). Energy differences between many-body eigenstates (dot chemical potentials) of the Anderson model and sketch of a COT process (a) and a SET process (b). (c) More detailed sketch of a PT process, giving rise to a resonance at $\Delta=$ $U / 2$ above the SET onset. Filled (unfilled) circles indicate real (virtual) occupation.
Experimentally, the dot energies can be controlled linearly by the gate voltage $V_{g}$. This produces the characteristic Coulomb diamond figures in differential conductance color maps as function of gate and bias voltage [1]. Since the resonance condition (1) for PT depends on the average of the two dot energies $E_{\sigma}$ the PT lines have the same slope as SET resonances. To distinguish the PT and SET resonances a consistent calculation of their transport signature is thus imperative.

Transport calculation.-The pair tunneling resonance is visible in the transport spectrum when the charging energy is larger than both the tunnel rate $\Gamma$ and temperature $T$, and originates from coherent two-electron processes which become important with increasing $\Gamma$. We can address the interesting regime $U>T>\Gamma$ using the real-time transport theory [8], which allows systematic treatment of processes beyond lowest order in $\Gamma$, while accounting for the competition of single- and two-electron processes, essential at the PT resonance. The central task is to calculate the stationary reduced density matrix of the dot in the basis of
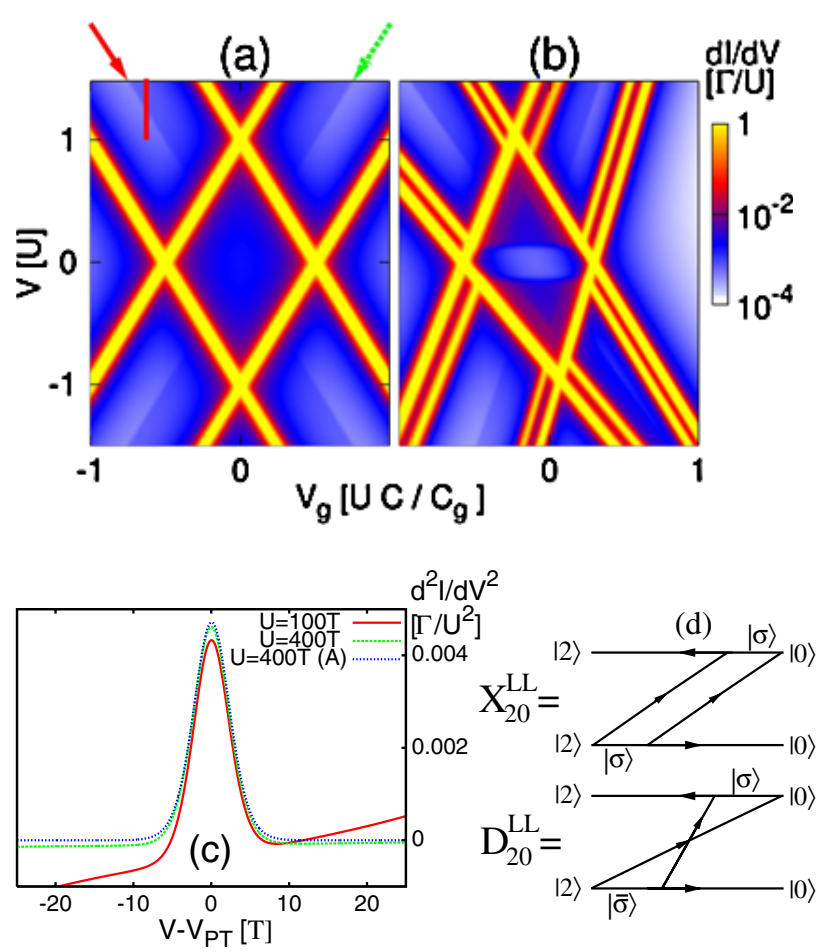

FIG. 2 (color online). (a) $d I / d V$ vs $V, V_{g}$ plotted on logarithmic color scale for $\Gamma_{L}=\Gamma_{R}=\Gamma, U=100 T=2000 \Gamma$ and $C_{R}=C_{L}=100 C_{g}$. (b) Same as (a) except for the finite Zeeman splitting by $h=15 T$ and asymmetric capacitances $C_{L}=0.7 C_{R}=100 C_{g}$. In addition, the gate capacitance of state 2 is larger: $C_{g, 2}=1.176 C_{g}$. (c) $d^{2} I / d V^{2}$ vs $V$ around the PT threshold $V_{\mathrm{PT}}$ at $V_{g}=-0.625 U C / C_{g}\left(C=C_{L}+C_{R}+C_{g}\right)$, indicated by the red line in (a). This demonstrates the scaling with $1 / U^{2}$ and the agreement with the analytic expression Eq. (3) marked by (A). (d) The two independent Keldysh diagrams contributing to the pair tunneling resonance. A sum over $\sigma$ is assumed and $\bar{\sigma}$ denotes the opposite spin projection of $\sigma$. 
many-body eigenstates, which is done using a kinetic (master) equation. From this density matrix the current flowing out of reservoir $r, I_{r}$, can be calculated. The required transport rates are calculated perturbatively up to second order in $\Gamma$ and are given by the analytic expressions which we derived for a general model in Ref. [9]. Importantly, all coherent one- and two-electron processes are included and the Coulomb interaction $U$ is treated nonperturbatively.

Figures 2(a) and 2(b) show the differential conductance as a function of gate and bias voltage, where in (b) the spindegeneracy is lifted by an applied magnetic field. SET processes give rise to the strong yellow lines which cross at zero bias at the charge degeneracy points. In (b) additional gate-independent steps show up in the $N=$ 1 Coulomb blockade region, indicating the onset of inelastic cotunneling leading to occupation of the excited spinstate. Subsequent relaxation of this state by SET gives rise to additional gate-dependent lines in the Coulomb blockade region, as described, e.g., in Refs. [10,11]. Clearly separated from these other resonances, the PT resonance associated with an electron pair tunneling onto (off) the dot appears as a gate-dependent step inside the SET region, indicated by a red solid (green dotted) arrow in (a). Considering the resonance position and its voltage dependence only, the PT could be mistaken for a weak SET process involving an excited state of the $N=1$ charge state with energy $\Delta=U / 2$ relative to the $N=1$ ground state. However, the resonance shape provides one crucial clue to the identification as PT: in Fig. 2(c) we show a bias trace along the red vertical line indicated in Fig. 2(a), where it is seen that the PT resonance appears as a peak in $d^{2} I / d V^{2}$, rather than in $d I / d V$ as for SET. The reason is that, analogous to inelastic cotunneling, the number of electron states in the reservoirs available for a PT process is proportional to the bias voltage [2]. Another clear way to separate PT and SET resonances is that the PT resonance exhibits no Zeeman splitting; see Fig. 2(b). The physical reason is that the tunneling of a pair involves a transition between states with the same spin $(N=0,2$ electron singlets), expressed by the appearance of the sum $E_{\uparrow}+$ $E_{\downarrow}$ in Eq. (1). Since PT relies on an empty or doubly occupied initial state, the resonances do not continue down into the linear transport regime, making PT an $e x$ clusively nonequilibrium effect. Additionally, in contrast to the magnetic field excitations, the PT resonances do not continue horizontally into the $N=1$ Coulomb blockade region as inelastic cotunneling steps. In cases which deviate from the simplest capacitive model, the PT resonance shows an additional distinct property. In Fig. 2(b) the capacitances associated with the left and right leads were chosen unequal and the doubly occupied state has a larger gate-coupling (this might happen if the many-body wave function of the doubly occupied state is localized closer to the gate electrode, or is less screened by the source and drain electrodes [12]). This causes the diamond to be both tilted and skewed, but the PT resonance can still be found by taking the average bias voltage positions [cf. (1)] of the $N=0 \leftrightarrow N=1$ and $N=1 \leftrightarrow N=2$ ground state transitions, respectively.

The precise amplitude and shape of the PT peak are also crucial for its correct identification. Here an analytic expression for the peak is helpful, which can be obtained for $U \gg T, \Gamma, h$. For this we focus on the upper left SET region in Figs. 2(a) and 2(b) $(V>0)$ where the PT involves the transition $|0\rangle \rightarrow|2\rangle$ with both electrons tunneling onto the dot from the left reservoir. Deep in the SET region the SET rates are constant, while all second order rates except the pair tunneling are approximately linear in bias voltage. Here the PT peak is given by

$$
\frac{d^{2} I_{L}^{\mathrm{PT}}}{d V^{2}}=2\left(\frac{\Gamma_{R}}{2 \Gamma_{L}+\Gamma_{R}}\right)^{2} \frac{d^{2}}{d V^{2}} W_{20}^{\mathrm{LL}} .
$$

There are only two independent terms in the perturbation expansion which contribute to the nonlinear voltage dependence of the rate for tunneling with an electron pair from the left reservoir, $W_{20}^{\mathrm{LL}}=\operatorname{Re}\left(X_{20}^{\mathrm{LL}}+D_{20}^{\mathrm{LL}}\right)$. These terms correspond to the real-time diagrams [8] shown in Fig. 2(d). The corresponding integrals can be solved analytically [9] without the need for ad hoc regularization. Equation (2) shows that the peak in the derivative of the differential conductance directly maps out the energy dependence of the pair tunneling rate. Neglecting all terms not contributing to the PT peak in the second derivative, assuming equal capacitances to the left and right reservoirs, we are left with

$$
\begin{aligned}
\frac{d^{2} I_{L}^{\mathrm{PT}}}{d V^{2}}= & \frac{4 \Gamma_{L} \Gamma_{R}}{\pi T U^{2}} \frac{\Gamma_{L} \Gamma_{R}}{\left(2 \Gamma_{L}+\Gamma_{R}\right)^{2}} F\left(\frac{E_{\uparrow}+E_{\downarrow}+U-V}{T}\right) \\
& \times \sum_{s= \pm} \frac{1}{1+s h / U}\left(1+\frac{1}{1+s h / U}\right) .
\end{aligned}
$$

The resonance condition (1) is seen in the argument of the function $F(x)=d^{2}(x b(x)) / d x^{2}=\left(\frac{x}{4} \operatorname{coth} \frac{x}{2}-\frac{1}{2}\right) / \sinh ^{2}\left(\frac{x}{2}\right)$, with $b(x)=1 /\left(e^{x}-1\right)$ being the Bose function and $F(0)=1 / 6$.

It is of interest to compare the PT with the inelastic COT, which is theoretically and experimentally well-studied. In general the PT and COT peaks are of comparable magnitude and their dependence on $T$ and $U$ are the same, implying that the PT and COT should be simultaneously experimentally accessible. A first difference is that the PT peak is completely insensitive to relaxation processes, since it involves a transition between ground states in different charge sectors. In contrast, the COT resonance consists of two parts. The part which remains even in the limit where the voltage dependence of the occupations is negligible (relaxation faster than the electron cotunneling and/or asymmetric junction $\Gamma_{L, R} \gg \Gamma_{R, L}$ ), is given by

$$
\frac{d^{2} I_{L}^{\mathrm{COT}}}{d V^{2}}=\frac{8 \Gamma_{L} \Gamma_{R}}{\pi T U^{2}} F\left(\frac{h-V}{T}\right) .
$$


This expression is valid at the particle hole symmetric point (center of the $N=1$ Coulomb blockade regime) in the limit $U \gg h>T>\Gamma$ and $V>0$. For slow relaxation and $\Gamma_{L} \approx \Gamma_{R}$ the COT peak height is additionally increased and also an asymmetry between $V>h$ and $V<$ $h$ appears due to the voltage dependence of the occupations [13]. For very small fields and $\Gamma_{L}=\Gamma_{R}$, the COT peak height is seen to be given by $9 / 2$ times that of the PT peak. A second difference is that the PT peak is strongly suppressed for very asymmetric tunnel rates, proportional to the square of the smaller coupling and, characteristically, the forward and reverse bias PT peak-heights differ by a factor 4 , in contrast to the COT peaks which remain symmetric. Finally, although a magnetic field does not cause a Zeeman splitting of the PT peak, Eq. (3), it does increase the peak height, in contrast to the COT peak, Eq. (4), where the situation is reversed: the peak height is independent of the field, while the position $(V=h$, independent of $V_{g}$ ) is shifted linearly.

Quantum dots with comparable level spacing and charging energy are especially well suited for observation of PT since the background SET current is featureless. In the case of a dense excitation spectrum, e.g., due to coupling to a localized vibrational mode, additional PT resonances complicate a high-resolution spectroscopic analysis. Extending the calculations of the nonequilibrium Anderson-Holstein model in Ref. [9] to finite $U$ we find that PT resonances give a signature similar to SET associated with a very weakly coupled second vibrational mode. Thus, the results presented here are of general importance for the analysis of models dealing with the various complexities of realistic quantum dots. In the noninteracting limit, $U=0$, the PT is completely suppressed. In general, PT involves interference between the two contributions (diagrams) shown in Fig. 2(d). This interference may be constructive, as in the Anderson model, or destructive in other models. Interestingly the relative sign of the interfering contributions is sensitive to the spin of the involved quantum dot states. For a generalized quantum dot model where the doubly occupied ground state is a triplet, we find that the PT is suppressed due to quantum interference and can be induced by a magnetic field. Finally, the PT also shows up in more complex transport quantities such as the current noise, which is sensitive to the effective charge transferred in the tunnel process (which is $\pm 2 e$ here). Extending the real-time approach to the calculation of shot-noise [14,15], including non-Markovian effects, we find that the PT resonance is associated with an increased Fano factor. The above examples serve to illustrate that pair tunneling effects are of general importance for transport through nanosystems.

Discussion and conclusion.-In this Letter we have theoretically predicted a signature of coherent tunneling of electron pairs in the single-electron transport regime, for quantum dots with positive charging energies exceeding the broadening by thermal and quantum fluctuations of charge. Current low-temperature measurements can access such fine details in the first three derivatives of the current with respect to voltage without dropping below the noise level [12]. Also, many molecular and carbon nanotube devices couple strongly to the electrodes, making first and second order transport features comparable. We mention the possibility of measuring the charging energy by identifying the PT resonance and reading $U / 2$ off in the stability diagram, thus limiting the voltages one needs to apply. Alternatively, the pair tunneling resonance provides a consistency check on SET level assignments. On a general level, the results indicate the importance of complete perturbative treatment of nonequilibrium problems [9].

We acknowledge many stimulating discussions with $\mathrm{H}$. Schoeller, J. König, and F. Reckermann and the financial support from DFG SPP-1243, the NanoSci-ERA, the Helmholtz Foundation and the FZ-Jülich (IFMIT).

[1] L. Kouwenhoven, C. Marcus, P. McEuen, S. Tarucha, R. Westervelt, and N. Wingreen, in Mesoscopic Electron Transport, edited by K. Sohn, L. Kouwenhoven, and G. Schön (Kluwer, Dordrecht, 1997).

[2] J. Lambe and R. C. Jaklevic, Phys. Rev. 165, 821 (1968).

[3] S. DeFranceschi, S. Sasaki, J. M. Elzerman, W. G. van der Wiel, S. Tarucha, and L. P. Kouwenhoven, Phys. Rev. Lett. 86, 878 (2001).

[4] E. Sela, H.-S. Sim, Y. Oreg, M.E. Raikh, and F. von Oppen, Phys. Rev. Lett. 100, 056809 (2008).

[5] J. Koch, M. E. Raikh, and F. von Oppen, Phys. Rev. Lett. 96, 056803 (2006).

[6] F. W. J. Hekking, L. I. Glazman, K. A. Matveev, and R. I. Shekhter, Phys. Rev. Lett. 70, 4138 (1993).

[7] P. S. Cornaglia and D. R. Grempel, Phys. Rev. B 71, 245326 (2005).

[8] J. König, H. Schoeller, and G. Schön, Phys. Rev. Lett. 76, 1715 (1996).

[9] M. Leijnse and M. R. Wegewijs, Phys. Rev. B 78, 235424 (2008); In Fig. 1(a) of this paper the PT resonance, although present, is not resolved with the color scale used.

[10] V. N. Golovach and D. Loss, Phys. Rev. B 69, 245327 (2004).

[11] R. Schleser, T. Ihn, E. Ruh, K. Ensslin, M. Tews, D. Pfannkuche, D.C. Driscoll, and A.C. Gossard, Phys. Rev. Lett. 94, 206805 (2005).

[12] E. A. Osorio, K. O’Neill, M. R. Wegewijs, N. StuhrHansen, J. Paaske, T. Bjørnholm, and H. S. van der Zant, Nano Lett. 7, 3336 (2007).

[13] J. Paaske, A. Rosch, and P. Wölfle, Phys. Rev. B 69, 155330 (2004).

[14] A. Thielmann, M. H. Hettler, J. König, and G. Schön, Phys. Rev. Lett. 95, 146806 (2005).

[15] J. Aghassi, M. Hettler, and G. Schön, Appl. Phys. Lett. 92, 202101 (2008). 